\title{
Towards a Unified Dynamical Theory of the Brownian Particle in an Ideal Gas
}

\author{
Domokos Szász and Bálint Tóth \\ Mathematical Institute of the Hungarian Academy of Sciences, P.O.B. 127, \\ H-1364 Budapest, Hungary
}

\begin{abstract}
We consider the trajectory $Q^{M}(t)$ of a Brownian particle of mass $M$ in an ideal gas of identical particles of mass 1 and of density 1 in equilibrium at inverse temperature 1 (the dynamics is uniform motion plus elastic collisions with the Brownian particle). Our theory, in dimension one, describes a variety of limiting processes - containing the Wiener process and the Ornstein-Uhlenbeck process - for $A^{-1 / 2} Q^{M(A)}(A t)$ depending on the asymptotic behaviour of $M(A)$. Part of the theory is hypothetical while another part relies upon known results. We also prove that, if $A^{\frac{1}{2}+\varepsilon} \ll M(A) \ll A$, then $A^{-1 / 2} Q^{M(A)}(A t)$ converges to a Wiener process whose variance is known from papers of Sinai-Soloveichik and of the present authors.
\end{abstract}

\section{Introduction}

Ed Nelson's classical notes about Brownian motion, N (1967), also containing an exciting historical account, stressed the necessity to derive Brownian motion from Hamiltonian principles. "The problem, or one formulation of it, is to deduce each of the following theories from the one below it:

\section{Einstein-Smoluchowski \\ Ornstein-Uhlenbeck \\ Maxwell-Boltzmann \\ Hamilton-Jacabi."}

His notes, in fact, show that "the Einstein-Smoluchowski theory is in a rigorous and strong sense the limiting theory of the Ornstein-Uhlenbeck theory." (Note that the mathematical model of the first theory is the Wiener process.)

The aim of the present paper is to realize the program for a Brownian particle interacting with an ideal gas of point particles. A rough outline of our theory is the following: if we start from a Gibbs equilibrium state, then the model contains a functional parameter describing the interdependence between the mass ratio of the Brownian particle and of the gas particles on one side and the space-time scaling of 
the trajectory of the Brownian particle on the other side. (In the understanding of modern statistical physics, the infinite Gibbs state corresponds to the MaxwellBoltzmann theory and it is known to describe the time-invariant state of a dynamics governed by the Hamilton-Jacobi formalism.) For simplicity suppose that this functional parameter, whose precise definition will be given in the next section, is of the form $f(A) \sim c A^{\gamma}$ as $A \rightarrow \infty(c>0)$. (Here larger values of $\gamma$ correspond to heavier Brownian particles.) Now our theory shows that by varying the functional parameter - or in this simple case $\gamma$ - one can obtain both the EinsteinSmoluchowski and the Ornstein-Uhlenbeck theories. Indeed, if $\frac{1}{2}<\gamma<1$ then and this is the main technical result of the paper - we get the Einstein-Smoluchowski theory, if $\gamma=1$, then the Ornstein-Uhlenbeck theory [in fact, this is an earlier result of Holley, $\mathrm{H}$ (1971) for $d=1$, and of Dürr-Goldstein-Lebowitz, D-G-L (1981) for $d \geqq 2$ ]. For $\gamma>1$ the theory is trivial, while for $0<\gamma \leqq \frac{1}{2}$ and $\gamma<0$ we expect the Einstein-Smoluchowski theory to hold, but so far we could not prove it. We also expect that in the case $\gamma=0$ the theory definitely depends on the spatial dimension of the system and on the value of $c$. (E.g. in dimension 1 computer results show a delicate picture, cf. Sect. 2c.) Besides its meaning for the general theory outlined above our result for $\frac{1}{2}<\gamma<1$ is hoped to open the way towards treating the very interesting case $M=$ const.

Section 2 describes the model, the theory and finally the result. The framework of its proof is given in Sect. 3. Sections 4 and 5 are technical, exposing the two main components of the proof: the analysis of the Markovized process and the construction of the coupling.

\section{The Theory and the Results}

\section{a) The Mathematical Model}

Since the results we prove are formulated in the one-dimensional case, for simplicity, we define the model for this case only.

A one-dimensional system of point particles consists of a tagged particle of mass $M$ (the Brownian particle) interacting with an infinite ideal gas of particles of mass 1 (light particles). The dynamics of the system is governed by the laws of classical mechanics assuming uniform motion plus elastic collisions between the Brownian particle and the light ones and no interaction among the light particles. (As far as the behavior of the Brownian particle is observed only, assuming elastic collisions among the light particles, too, would not change the picture.)

The collision rules are the following:

$$
V^{+}=\frac{M-1}{M+1} V^{-}+\frac{2}{M+1} v^{-}, \quad v^{+}=\frac{2 M}{M+1} V^{-}-\frac{M-1}{M+1} v^{-}
$$

or

$$
\Delta V=V^{+}-V^{-}=-\frac{2}{M+1}\left(V^{-}-v^{-}\right) ; \quad \Delta v=v^{+}-v^{-}=\frac{2 M}{M+1}\left(V^{-}-v^{-}\right),
$$

where $V^{ \pm}, v^{ \pm}$are the post- (pre-) collision velocties of the colliding Brownian respectively light particle. The most convenient is to describe our system as seen 
from the Brownian particle [the so-called "Münchhausen picture" cf. B (1788)]. In this picture the phase space is

$$
\mathfrak{X}=\mathbb{R} \times \Omega=\left\{x=(V, \omega): V \in \mathbb{R}, \omega=\left(q_{i}, v_{i}\right)_{i \in I} \in \Omega\right\},
$$

where $I$ is a countably infinite index set, $\Omega$ the set of locally finite countable point systems in $\mathbb{R} \times \mathbb{R}$. ( $V$ is the velocity of the Brownian particle, $\left(q_{i}, v_{i}\right)_{i \in I}$ are the coordinates - relative to the position of the Brownian particle - and the velocities of the light particles.) We say that $\omega$ is the environment seen by the Brownian particle. $\Omega$ is a polish space endowed with the natural $\sigma$-algebra $\mathscr{F}_{0}$ generated by counting functions on compact sets. The $\sigma$-algebra on $\mathfrak{X}$ is $\mathscr{F}=\mathscr{B} \times \mathscr{F}_{0}, \mathscr{B}$ being the Borelalgebra on $\mathbb{R}$. The system is distributed according to the Gibbs measure

$$
\mu^{M}(d(V, \omega))=d F^{M}(V) \cdot v(d \omega)
$$

with $v$ being the Poisson measure on $\left(\Omega, \mathscr{F}_{0}\right)$ with intensity $d x d F^{1}(v)$ and

$$
d F^{M}(V)=\sqrt{\frac{M}{2 \pi}} \exp \left(-\frac{M V^{2}}{2}\right) d V \quad(M>0) .
$$

More prosaically: the positions of the light particles follow a Poissonian point process of density $\varrho=1$, while the velocities of the particles are distributed according to independent, zero-mean Gaussians of variance equal to (mass) ${ }^{-1}$, i.e. Maxwellian velocity distributions at inverse temperature $\beta=1$.

Remark. The only essential parameter of the system is $M$, while $\varrho$ and $\beta$ enter trivially into the theory.

Denote by $S_{t}^{M}$ the dynamics of the system. The following two facts are assumed to be known:

a) for each $M$ there exists a set $\mathfrak{X}^{M} \subset \mathfrak{X}$ of $\mu^{M}$-measure 1 on which the maps $S_{t}^{M}$ are well defined for any $t \in \mathbb{R}$, and $S_{t+s}^{M}=S_{t}^{M} \circ S_{s}^{M}$. (The equilibrium dynamics exists with probability 1.)

b) the group of transformations $S_{t}^{M}: \mathfrak{X}^{M} \rightarrow \mathfrak{X}^{M}$ preserves the measure $\mu^{M}$. (The evolution of the system, as seen from the Brownian particle, is stationary.) Warning: for different $M$ 's we have different dynamical systems $\left(\mathfrak{X}^{M}, \omega^{M}, S_{t}^{M}\right)$. The random variables to be introduced below are defined on different probability spaces, depending on $M$. But, as we are interested in asymptotic laws, this fact is not at all disturbing.

We shall use the notations

$$
\begin{aligned}
& V(x)=V \quad \text { and } \quad \omega(x)=\omega \text { iff } \quad x=(V, \omega) \in \mathfrak{X}, \\
& V_{t}^{M}(x)=V\left(S_{t}^{M} x\right), \quad x \in \mathfrak{X}^{M}, \\
& Q_{t}^{M}(x)=\int_{0}^{t} V_{s}^{M}(x) d s, \quad x \in \mathfrak{X}^{M} .
\end{aligned}
$$

\section{b) An Intermezzo: Simple Facts About the Ornstein-Uhlenbeck Process}

Throughout this paper $W_{t}^{(\sigma)}$ will denote a Wiener process of variance $\sigma^{2}$ with $W_{0}^{(\sigma)}=0$ and for berevity let $W_{t}=W_{t}^{(1)}$. 
The diffusion process $\eta_{t}$ satisfying the stochastic differential equation

$$
d \eta_{t}=-\gamma \eta_{t} d t+\sqrt{D} d W_{t}
$$

is called an Ornstein-Uhlenbeck (velocity) process. If $\eta_{0}$ is distributed according to the Gaussian law with mean 0 and variance $(2 \gamma)^{-1} D$, then $\eta_{t}$ is a stationary GaussMarkov process and its generator is

$$
L=-\gamma x \frac{d}{d x}+D \frac{1}{2} \frac{d^{2}}{d x^{2}} .
$$

The integral process

$$
\xi_{t}=\int_{0}^{t} \eta_{s} d s
$$

is called the Ornstein-Uhlenbeck position process. We shall use these processes with the following choice of parameters

$$
\gamma=\frac{4}{m} \sqrt{\frac{2}{\pi}}, \quad D=\frac{8}{m^{2}} \sqrt{\frac{2}{\pi}},
$$

and we will use the notations $\eta_{t}^{(m)}$ and $\xi_{t}^{(m)}$ for them ( $m$ is a positive constant). It is worth mentioning that if $\gamma \rightarrow \infty, D \rightarrow \infty$ in such a way that $D \gamma^{-2} \rightarrow \sigma^{2} \in \mathbb{R}^{+}$, then the Ornstein-Uhlenbeck position process $\xi_{t}$ converges in distribution to a Wiener process $W^{(\sigma)}$ [see N (1967)]. Thus

$$
\xi_{t}^{(m)} \Rightarrow W_{t}^{(\underline{\underline{\sigma})}} \quad, \quad \text { as } \quad m \rightarrow 0 .
$$

with $\underline{\sigma}^{2}=\sqrt{\frac{\pi}{8}}$.

c) The Scaling and the Theory

Our final aim is to give a complete asymptotic description of the random processes

$$
\frac{Q_{A t}^{M(A)}}{\sqrt{A}} \text { as } A \rightarrow \infty .
$$

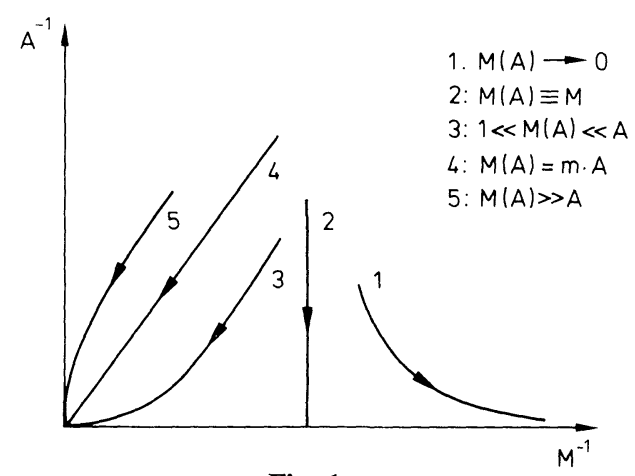

Fig. 1 
Observe that the space-time scaling is the usual diffusion one which is used, for example, to obtain a Wiener limiting process for random walks. $M(A)$ expresses the dependence of the mass ratio of the Brownian particle versus the light ones on the parameter $A$ figuring in the space-time scaling. The important types of dependence to be considered are illustrated on Fig. 1.

Throughout this paper $f(A) \ll g(A)$ will have the precise meaning $f(A)=o(g(A))$ as $A \rightarrow \infty$.

Several cases have already been clarified but the picture is still far from complete. Here we list the most important existing results following a logical order rather than the chronological one.

(A) For $M(A) \equiv 1$, that is: the Brownian particle is identical with the light ones, Harris (1965) and Spitzer (1969) proved that

$$
\frac{Q_{A}^{1}}{\sqrt{A}} \Rightarrow W^{(\bar{\sigma})}, \quad \bar{\sigma}^{2}=\sqrt{\frac{2}{\pi}} .
$$

(Throughout this paper $\Rightarrow$ stands for weak convergence on $C[0, \infty)$, the space of continuous functions or on $D[0, \infty)$, that of right continuous functions without a second order discontinuity on $[0, \infty)$.)

(B) For arbitrary fixed mass $M(A) \equiv M$ Sinai and Soloveichik (1986) and the present authors [Sz-T (1986)] showed that

$$
\sqrt{\frac{\pi}{8}} t=\underline{\sigma}^{2} t \leqq \varlimsup_{\overline{A \rightarrow \infty}} \mathbb{E}\left(\frac{Q_{A t}^{(M)}}{\sqrt{A}}\right)^{2} \leqq \bar{\sigma}^{2} t=\sqrt{\frac{2}{\pi}} t .
$$

Computer results [by D-O-R (1985) and by Sinai's group S (1986)] suggest the following picture: for every $M$

$$
\sigma_{M}^{2}=\frac{1}{t} \lim _{A \rightarrow \infty} \mathbb{E}\left(\frac{Q_{A t}^{(M)}}{\sqrt{A}}\right)^{2}
$$

exists and by (2.3), of course, $\underline{\sigma} \leqq \sigma_{M} \leqq \bar{\sigma}$. Moreover, the dependence of $\sigma_{M}$ on $M$ is illustrated on Fig. 2.

(C) From the proofs of Sz-T (1986) it is easy to see that, in (2.3), the upper bound holds for an arbitrary scaling functional $M(A)$ while the lower bound holds whenever $M(A)=o(A)$.

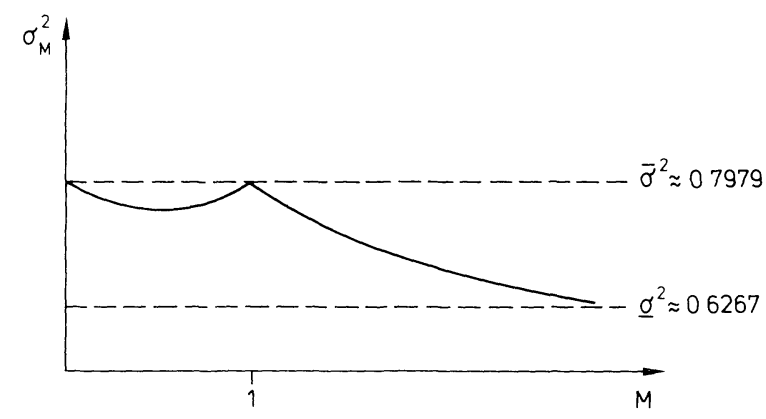

Fig. 2 
(D) For $M(A)=m \cdot A, m \in(0, \infty)$ Holley (1971) proved that

$$
\sqrt{A} V_{A \cdot}^{(m A)} \Rightarrow \eta^{(m)} ; \quad \frac{Q_{A}^{(m A)}}{\sqrt{A}} \Rightarrow \xi^{(m)} .
$$

Important Remark. The results (B) and (D) can be linked by observing that [cf. (2.2)]

$$
\xi^{(m)} \Rightarrow W^{(\bar{\sigma})} \quad \text { as } \quad m \rightarrow 0 .
$$

On the basis of the aforementioned results we expect the following complete asymptotic picture [the dimension is still 1; the multidimensional case requires further elaboration since the model has an additional parameter: the size of the Brownian particle, cf. D-G-L (1981)].

1. Case $M(A) \rightarrow 0$.

$$
\frac{Q_{A}^{M(A)}}{\sqrt{A}} \Rightarrow W^{(\bar{\sigma})}
$$

2. Case $M(A)=M$.

$$
\frac{Q_{A}^{M}}{\sqrt{A}} \Rightarrow T^{M} \quad \underline{\sigma} \leqq \sigma_{M} \leqq \bar{\sigma},
$$

where $T^{M}, M>0$ are random processes with stationary increments and with asymptotic variance $\sigma_{M}^{2}$.

It is an extremely intriguing question whether, in general, $T^{M}$ is a Wiener process or not. Computer results by Sinai's group (S (1986)) suggest that, for a general $M$, it is not.

We know that $T^{1}=W^{\bar{\sigma}}$, while simulations support that $\sigma_{M} \rightarrow \underline{\sigma}$ as $M \rightarrow \infty$ and $\sigma_{M} \rightarrow \bar{\sigma}$ as $M \rightarrow 0$ [the result for $M \equiv 1$ was proved in $\mathrm{H}$ (1965) and S (1969) while the bounds on the variances were given in S-S (1986) and Sz-T (1986)].

3. Case $1 \ll M(A) \ll A$.

$$
\frac{Q_{A}^{M(A)}}{\sqrt{A}} \Rightarrow W^{(\sigma)}
$$

4. Case $M A)=m A$.

$$
\frac{Q_{A}^{M(A)}}{\sqrt{A}} \Rightarrow \xi^{(m)}
$$

where $\xi^{(m)}$ is introduced in Sect. $2 b$. This convergence was proved in $\mathrm{H}(1971)(d=1)$ and in D-G-L (1981) $(d \geqq 1)$. For $m \rightarrow 0$, (2.2) holds. For $m \rightarrow \infty, \xi^{(m)} \Rightarrow 0$.

5. Case $M(A) \gg A$.

$$
\frac{Q_{A \cdot}^{M(A)}}{\sqrt{A}} \Rightarrow 0 \text { (trivial) }
$$


(Of course, the problem is not trivial if we allow spatial rescalings different from $A^{1 / 2}$; its determination is a question of independent interest.)

In the present note we make one further step in completing the picture sketched above, by proving the following

Theorem 2.1. If $A^{\frac{1}{2}+\varepsilon} \ll M(A) \ll A \quad(\varepsilon>0)$, then

$$
\frac{Q_{A}^{M(A)}}{\sqrt{A}} \Rightarrow W^{(\sigma)}
$$

\section{The Framework of the Proof}

The first - and in a sense principal - difficulty in the dynamics of the Brownian particle is the non-Markovity of its motion. Indeed, light particles between their first and last collisions with the Brownian one carry information on past collisions in a complicated way. Nonetheless it is a natural idea to consider a Markov process whose evolution mimics the physical process; this Markov process, of course, disregards recollisions that could spoil its Markovity. This Markov version can help both on an intuitive level to give a feeling of what the mechanical process is like and on a technical level, too, if we can construct a good coupling between the mechanical and the Markov processes. In our knowledge, this idea was first used in a rigorous argument by Holley (1971) [cf. case (D), Sect. 2c] and our proof is also a realization of this strategy [other variants of this idea can be found in D-G-L (1981) and G-G (1986)].

Let us first construct a family of Markov processes $\widetilde{V}_{t}^{M}, M>0$ closely related to the mechanical velocity processes $V_{t}^{M}$. In words, $\tilde{V}_{t}^{M}$ 's are defined as follows: we imagine that the environment is recreated, after each collision, corresponding to the time-invariant distribution $v$. Thus the Markovian velocity process $\widetilde{V}_{t}^{M}$ is a pure jump process on $\mathbb{R}$ with jump rates

$$
\text { Rate }\left(V \rightarrow \frac{M-1}{M+1} V+\frac{2}{M+1} v\right)=\frac{1}{\sqrt{2 \pi}} e^{-\frac{v^{2}}{2}|V-v| d v}
$$

In the actual coordinates the jump rates are

$$
R^{M}(x, y) d y=\frac{1}{\sqrt{2 \pi}}\left(\frac{M+1}{2}\right)^{2} \exp \left[-\frac{1}{2}\left(\frac{M+1}{2} y-\frac{M-1}{2} x\right)^{2}\right]|x-y| d y
$$

leading to the formal generator

$$
\begin{aligned}
\left(\widetilde{G}_{M} \phi\right)(x) & =\frac{1}{\sqrt{2 \pi}} \int d y e^{-\frac{y^{2}}{2}}|y-x|\left\{\phi\left(\frac{M-1}{M+1} x+\frac{2}{M+1} y\right)-\phi(x)\right\} \\
& =\frac{1}{\sqrt{2 \pi}}\left(\frac{M+1}{2}\right)^{2} \int d y e^{-\frac{1}{2}\left(\frac{M+1}{2} y-\frac{M-1}{2} x\right)^{2}}|y-x|\{\phi(y)-\phi(x)\} .
\end{aligned}
$$


It is easily seen that, for $\phi$ and $\psi$ belonging to a sufficiently large class of functions,

$$
\int d F^{M}(x) \phi(x)\left(\widetilde{G}_{M} \psi\right)(x)=\int d F^{M}(x)\left(\widetilde{G}_{M} \phi\right)(x) \psi(x) .
$$

Thus, the Markov processes $\widetilde{V}_{t}^{M}$ conditioned to the initial distributions $d F^{M}(x)$ are stationary and reversible. (We shall see soon that they are ergodic, too.)

Now the program consists of two parts:

(i) a study of the induced position processes,

$$
\widetilde{Q}_{t}^{M}=\int_{0}^{t} \widetilde{V}_{s}^{M} d s
$$

(ii) construction of a good coupling for $Q_{t}^{M}$ and $\widetilde{Q}_{t}^{M}$, i. e. a realization of $Q_{t}^{M}$ and $\widetilde{Q}_{t}^{M}$ on the same probability space, that satisfies

$$
\frac{1}{\sqrt{A}}\left(Q_{A t}^{M(A)}-\widetilde{Q}_{A t}^{M(A)}\right) \Rightarrow 0 \text { as } A \rightarrow \infty .
$$

Part (i) is executed in Sect. 4. Here the main result is

Theorem 3.1. (i) (fixed masses). For any fixed $M \in(0, \infty)$,

with

$$
\frac{\widetilde{Q}_{A}^{M}}{\sqrt{A}} \Rightarrow W^{\left(\tilde{\sigma}_{M}\right)}
$$

and

$$
\tilde{\sigma}_{M}^{2} \geqq \sqrt{1+\frac{1}{M}} \underline{\sigma}^{2}
$$

$$
\lim _{M \rightarrow \infty} \tilde{\sigma}_{M}^{2}=\underline{\sigma}^{2}
$$

(ii) (sublinearly increasing masses). If $1 \ll M(A) \ll A$, then

$$
\frac{\widetilde{Q}_{A}^{M(A)}}{\sqrt{A}} \Rightarrow W^{(\tilde{\sigma})} .
$$

In fact, our methods give the following complete asymptotic characterization of the induced position processes $\widetilde{Q}_{t}^{M}$ (the reader is encouraged to compare it with the analogous picture formulated for $Q_{t}^{M}$ in the preceding section).

1. Case $M(A) \rightarrow 0, \frac{\widetilde{Q}_{A}^{M(A)}}{\sqrt{A}}$ is not tight.

2. Case $M(A)=M, \frac{\widetilde{Q}_{A}^{M(A)}}{\sqrt{A}} \Rightarrow W^{\left(\tilde{\sigma}_{M}\right)}$,

with $\tilde{\sigma}_{M}^{2} \sim M^{-1 / 2}$ for $M \rightarrow 0$ and $\tilde{\sigma}_{M}^{2} \rightarrow \underline{\sigma}^{2}$ as $M \rightarrow \infty$.

3. Case $1 \ll M(A) \ll A, \quad \frac{\widetilde{Q}_{A}^{M(A)}}{\sqrt{A}} \Rightarrow W^{(\tilde{\tilde{\sigma}})}$. 
4. Case $M(A)=m A, \quad \frac{\widetilde{Q}_{A}^{M A)}}{\sqrt{A}} \Rightarrow \xi^{m}$

5. Case $M(A) \gg A, \quad \frac{\widetilde{Q}_{A}^{M(A)}}{\sqrt{A}} \Rightarrow 0$

Cases 1-3 follow from Theorem 3.1; Case 4 is proved in H (1981); Case 5 is trivial.

As to part (ii), so far we could only do the coupling under the assumption $M(A) \gg A^{\frac{1}{2}+\varepsilon}$. The slower $M(A)$ increases the stronger the influence of the recollisions for the mechanical process is and the estimates required for the coupling become harder and harder.

Remarks. We expect that further progress can be achieved soon in this circle of ideas.

a) For $1 \ll M(A) \ll A^{\frac{1}{2}+\varepsilon}$, we hope that some sophisticated refinement of the coupling argument may work.

b) For $M(A)=M \in(0, \infty)$, naturally, a "good coupling" cannot be realized, but our estimates for $\tilde{\sigma}_{M}$ may be of some use in estimating $\sigma_{M}$ for large $M$.

\section{Study of the Markovized Process}

As a preparation for the proof of Theorem 3.1 we give a detailed analysis of the $L_{2}$-properties of the generators $\widetilde{G}_{M}$. We consider the Hilbert spaces $\mathscr{H}^{M}=L_{2}\left(\mathbb{R}, \sqrt{\frac{M}{2 \pi}} e^{-\frac{M x^{2}}{2}} d x\right) ; \mathscr{H}=\mathscr{H}^{1}$ with the scalar products denoted by $(,)_{M}$ and (,) respectively. The generators $\widetilde{G}_{M}$ have to be considered as (unbounded) operators on the Hilbert spaces $\mathscr{H}^{M}$, but it is more convenient to transform all operators into one standard Hilbert space, $\mathscr{H}$, by the unitary isomorphisms

$$
U^{M}: \mathscr{H}^{M} \rightarrow \mathscr{H} \quad\left(U^{M} \phi\right)(x)=\phi\left(\frac{x}{\sqrt{M}}\right) .
$$

The images of the generators under these isomorphisms are

$$
\begin{gathered}
U^{M} \widetilde{G}_{M}\left(U^{M}\right)^{-1} \stackrel{\text { def }}{=} G_{M}, \\
\left(G_{M} \phi\right)(x)=\frac{1}{\sqrt{2 \pi}} \int d y e^{-\frac{y^{2}}{2}} \mathscr{K}_{M}(x, y)\{\phi(y)-\phi(x)\} \\
=\frac{1}{\sqrt{2 \pi}} \int d y e^{-\frac{y^{2}}{2}} \mathscr{K}_{M}(x, y) \phi(y)-\gamma\left(\frac{x}{\sqrt{M}}\right) \phi(x)=\left(K_{M}-\Gamma_{M}\right) \phi(x),
\end{gathered}
$$


where

$$
\begin{gathered}
\mathscr{K}_{M}(x, y)=\left(\frac{M+1}{2 \sqrt{M}}\right)^{2} \exp -\frac{1}{2}\left[\left(\frac{M-1}{2 \sqrt{M}}\right)^{2} y^{2}-2 \frac{M-1}{2 \sqrt{M}} \cdot \frac{M+1}{2 \sqrt{M}} x y\right. \\
\left.+\left(\frac{M-1}{2 \sqrt{M}}\right)^{2} x^{2}\right]|x-y|, \\
\gamma(x)=\frac{1}{\sqrt{2 \pi}} \int d y e^{-\frac{y^{2}}{2}}|x-y| \text { and } \gamma\left(\frac{x}{\sqrt{M}}\right)=\frac{1}{\sqrt{2 \pi}} \int d y e^{-\frac{y^{2}}{2}} \mathscr{K}_{M}(x, y) .
\end{gathered}
$$

Here $K_{M}$ denotes the integral operator on $\mathscr{H}$ having $\mathscr{K}_{M}$ as kernel function and $\Gamma_{M}$ denotes the multiplication operator by the function $\gamma\left(\frac{\cdot}{\sqrt{M}}\right) \cdot \gamma(x)$ can be computed explicitly. Essential facts are that: 1) it is continuous; 2) it has a global minimum with value $\sqrt{\frac{2}{\pi}}$ at $x=0$ and 3 ) it grows linearly for large values of $x$. It is easy to see that $K_{M}$ is a self adjoint Hilbert-Schmidt operator, with Hilbert-Schmidt norm

$$
\left\|K_{M}\right\|_{\mathrm{H}-\mathrm{S}}^{2}=\frac{(M+1)^{4}}{8 M^{3}} .
$$

(In fact, it is of trace class $I_{p}$ for $p>\frac{2}{3}$.) Consequently the generators $G_{M}$ are selfadjoint on a common domain

$$
D=\{\phi \in \mathscr{H} \mid x \cdot \phi \in \mathscr{H}\} .
$$

The following identity is easy to check:

$$
\left(\phi, G_{M} \phi\right)=-\frac{1}{4 \pi} \cdot \iint d x d y e^{-\frac{x^{2}+y^{2}}{2}} \mathscr{K}_{M}(x, y)\{\phi(y)-\phi(x)\}^{2} .
$$

$G_{M}$ being the generator of a self-adjoint strongly continuous contraction semigroup, the fact that it is non-positive is not surprising. From the preceding formula one can also see that 0 is a nondegenerate eigenvalue of $G_{M}$, thus the stationary Markov process $\widetilde{V}_{t}^{M}$ is also ergodic [see $\mathrm{N}(1964)$ ].

It is worth mentioning (although it is of no use for our purposes) that

$$
M G_{M} \stackrel{\text { st. res }}{\longrightarrow} L \text { as } M \rightarrow \infty,
$$

where $\stackrel{\text { st. res }}{\longrightarrow}$ stands for convergence in the strong resolvent sense and $L$ is the generator of the standard Ornstein-Uhlenbeck velocity process $\eta_{t}^{(1)}$ (cf. Sect. 2b) This fact was crucial in the proofs of H (1971) and D-G-L (1981). Knowing the spectrum of $L$ (nondegenerate eigenvalues at the points $-4 \cdot \sqrt{\frac{2}{\pi}} n, n \in \mathbb{N}$ ) one can guess that the operators $G_{M}$ have gaps of order $\frac{\text { const }}{M}$ in their spectrum at 
left to the eigenvalue 0 , but, unfortunately, strong-resolvent convergence does not imply convergence of the spectrum. We shall prove in another way the following.

Lemma 4.1 (Gap-Lemma). If $1 \leqq M<\infty$,

$$
\sigma\left(G_{M}\right) \cap\left(-\frac{2}{M+1} \sqrt{\frac{2}{\pi}}, 0\right)=\emptyset .
$$

(In the present lemma $\sigma$ and $\sigma_{\text {ess }}$ denote spectrum and essential spectrum, respectively.)

Proof. $G_{M}$ being a compact perturbation of $-\Gamma_{M}$, by Theorem XIII.14 of R-S (1978) we have

$$
\sigma_{\mathrm{ess}}\left(G_{M}\right)=\{-\gamma(x): x \in \mathbb{R}\}=\left(-\infty,-\sqrt{\frac{2}{\pi}}\right]
$$

consequently it is enough to show that there are no eigenvalues in the interval $\left(-\frac{2}{M+1} \sqrt{\frac{2}{\pi}}, 0\right)=I_{M}$. Soon we shall prove

Lemma 4.2. If $\notin \sigma_{\mathrm{ess}}$ and

$$
G_{M} \phi=\lambda \phi,
$$

then $\phi$ is differentiable with $\phi^{\prime} \in \mathscr{H}$ and

$\frac{M-1}{M+1}\left(\phi^{\prime}, G_{M} \phi^{\prime}\right)+\frac{1}{2}\left(\phi, G_{M} \phi\right)=\frac{1}{\sqrt{2 \pi}} \cdot \int e^{-\frac{x^{2}}{2}}\left[\frac{2}{M+1} \gamma\left(\frac{x}{\sqrt{M}}\right)+\lambda\right] \phi^{\prime 2}(x) d x$.

This lemma plus the non-positivity of $G_{M}$ provide the desired result.

Proof of Lemma 4.2. For $\lambda>-\sqrt{\frac{2}{\pi}},(4.5)$ is equivalent to

$$
\phi(x)=\frac{\frac{1}{\sqrt{2 \pi}} \cdot \int d y e^{-\frac{y^{2}}{2}} \mathscr{K}_{M}(x, y) \phi(y)}{\gamma\left(\frac{x}{\sqrt{M}}\right)+\lambda} .
$$

Differentiating the right-hand side with respect to $x$ after some tedious calculations, using Schwartz's inequality one finds bounds on $\phi^{\prime}(x)$, showing that, indeed, $\phi^{\prime} \in \mathscr{H}$. The details are standard and we omit them. More illuminating is the proof 
of the identity (4.6). To do this we have to introduce - besides $\mathscr{K}_{M}$ - two auxiliary kernels

$$
\begin{aligned}
\mathscr{K}_{M}^{1}(x, y)= & \left(\frac{M+1}{2 \sqrt{M}}\right)^{2} \exp -\frac{1}{2}\left[\left(\frac{M-1}{2 \sqrt{M}}\right)^{2} x^{2}-2 \frac{(M-1)(M+1)}{(2 \sqrt{M})^{2}} x y\right. \\
& \left.+\left(\frac{M-1}{2 \sqrt{M}}\right)^{2} y^{2}\right] \cdot \operatorname{sign}(x-y) \\
\mathscr{K}_{M}^{2}(x, y)= & \left(\frac{M+1}{2 \sqrt{M}}\right)^{2} \exp -\frac{1}{2}\left[\left(\frac{M-1}{2 \sqrt{M}}\right)^{2} x^{2}-2 \frac{(M-1)(M+1)}{(2 \sqrt{M})^{2}} x y\right. \\
& \left.+\left(\frac{M-1}{2 \sqrt{M}}\right)^{2} y^{2}\right] \cdot \delta(x-y) .
\end{aligned}
$$

The following identities hold:

$$
\begin{aligned}
\frac{\partial}{\partial x}\left[e^{-\frac{y^{2}}{2}} \mathscr{K}_{M}(x, y)\right]= & -\frac{M-1}{M+1} \cdot \frac{\partial}{\partial y}\left[e^{-\frac{y^{2}}{2}} \mathscr{K}_{M}(x, y)\right] \\
& +\frac{2}{M+1} e^{-\frac{y^{2}}{2}} \mathscr{K}_{M}^{1}(x, y)
\end{aligned}
$$

and

$$
\begin{aligned}
& \frac{\partial}{\partial x}\left[e^{-\frac{x^{2}+y^{2}}{2}} \mathscr{K}_{M}^{1}(x, y)\right] \\
& \quad=e^{-\frac{x^{2}+y^{2}}{2}}\left[-\frac{M+1}{4} \mathscr{K}_{M}(x, y)-\frac{M+1}{4 M} \cdot(x+y) \mathscr{K}_{M}^{1}(x, y)+\mathscr{K}_{M}^{2}(x, y)\right] .
\end{aligned}
$$

Now differentiating both sides of (4.5) with respect to $x$ and using (4.7), after a partial integration we find

$$
\begin{aligned}
& \frac{M-1}{M+1} \cdot \frac{1}{\sqrt{2 \pi}} \cdot \int d y e^{-\frac{y^{2}}{2}} \mathscr{K}_{M}(x, y)\left\{\phi^{\prime}(y)-\phi^{\prime}(x)\right\} \\
& \quad+\frac{2}{M+1} \cdot \frac{1}{\sqrt{2 \pi}} \int d y e^{-\frac{y^{2}}{2}} \mathscr{K}_{M}^{1}(x, y)\{\phi(y)-\phi(x)\} \\
& =\left(\frac{2}{M+1} \gamma\left(\frac{x}{\sqrt{M}}\right)+\lambda\right) \phi^{\prime}(x) .
\end{aligned}
$$

We take now scalar product of both sides of this identity, with $\phi^{\prime}$. After a new integration by parts in the second term/of the left-hand side, the equation takes the 
form

$$
\begin{aligned}
& \frac{M-1}{M+1} \cdot \frac{-1}{4 \pi} \cdot \iint d x d y e^{-\frac{x^{2}+y^{2}}{2}} \mathscr{K}_{M}(x, y)\left\{\phi^{\prime}(y)-\phi^{\prime}(x)\right\}^{2} \\
& \quad+\frac{1}{M+1} \cdot \frac{1}{2 \pi} \cdot \iint d x d y \frac{\partial}{\partial x}\left(e^{-\frac{x^{2}+y^{2}}{2}} \mathscr{K}_{M}^{1}(x, y)\right)\{\phi(y)-\phi(x)\}^{2} \\
& =\frac{1}{\sqrt{2 \pi}} \cdot \int d x e^{-\frac{x^{2}}{2}}\left[\frac{2}{M+1} \gamma\left(\frac{x}{\sqrt{M}}\right)+\lambda\right] \phi^{\prime 2}(x) .
\end{aligned}
$$

Using (4.8), (4.4) and observing that

$$
\begin{aligned}
& \iint d x d y e^{-\frac{x^{2}+y^{2}}{2}}(x+y) \mathscr{K}_{M}^{1}(x, y)\{\phi(y)-\phi(x)\}^{2} \\
& =\iint d x d y e^{-\frac{x^{2}+y^{2}}{2}} \mathscr{K}_{M}^{2}(x, y)\{\phi(y)-\phi(x)\}^{2}=0,
\end{aligned}
$$

we obtain exactly (4.6). Thus Lemma 4.2, and hence Lemma 4.1 are proved.

In the rest of this section we will be concerned with the subspace (of codimension 1)

$$
\mathscr{H}_{0}=\left\{\phi \in \mathscr{H} \mid \frac{1}{\sqrt{2 \pi}} \int d x e^{-\frac{x^{2}}{2}} \phi(x)=0\right\}
$$

orthogonal to the constant functions. As a consequence of the previous lemma the generators $G_{M}$ are invertible on this subspace and

In the sequel let

$$
\left\|\left.\left(M G_{M}\right)\right|_{\mathscr{H}_{0}} ^{-1}\right\| \leqq c=\sqrt{\frac{\pi}{2}} .
$$

One can easily show that

$$
\phi \in \mathscr{H}, \quad \phi(x)=x
$$

and, using (4.9) and (4.10)

$$
M G_{M} \phi \rightarrow-\frac{2}{\underline{\sigma}^{2}} \phi \quad \text { as } \quad M \rightarrow \infty
$$

$$
\left(M G_{M}\right)^{-1} \phi \rightarrow-\frac{\sigma^{2}}{2} \phi \quad \text { as } \quad M \rightarrow \infty .
$$

Relations (4.9), (4.10), and (4.11) will be the basic ingredients used in the calculational details of the forthcoming proof.

Proof of Theorem 3.1. The proof goes through a martingale approximation. The circle of ideas has been developed in G-L (1978), K-V (1986), D-G (1986). Our case (ii), however, is not covered by the results obtained there because we have a double array. In fact, the gap condition (4.9) will ensure the necessary momentum estimates uniformly in $M$.

Let

$$
\phi_{M} \in \mathscr{H}^{M} ; \quad \phi_{M}(x)=x, \quad U^{M} \phi_{M}=\frac{1}{\sqrt{M}} \phi
$$


Our position process is

$$
\widetilde{Q}_{t}^{M}=\int_{0}^{t} \tilde{V}_{s}^{M} d s=\int_{0}^{t} \phi_{M}\left(\tilde{V}_{s}^{M}\right) d s .
$$

Case (i): $\widetilde{Q}_{t}^{M}$ is an additive functional of an ergodic Markov process whose generator is invertible on the subspace orthogonal to the constant functions, thus the Theorem of G-L (1978) works. The limiting variance is

$$
\tilde{\sigma}_{M}^{2}=-2\left(\phi_{M}, \widetilde{G}_{M}^{-1} \phi_{M}\right)_{M}=-2\left(\phi,\left(M G_{M}\right)^{-1} \phi\right) .
$$

The lower estimate for $\tilde{\sigma}_{M}$ follows from straightforward calculations, while the asymptotics for $M \rightarrow \infty$ follows from (4.11).

Case (ii): Let

$$
\psi_{M} \in \mathscr{H}_{M}, \quad \psi_{M}=-\frac{\sigma^{2}}{2} M \widetilde{G}_{M} \phi_{M}
$$

We write the position process in the form

where

$$
Q_{t}^{M}=N_{t}^{M}+X_{t}^{M}+Y_{t}^{M}+Z_{t}^{M},
$$

$$
\begin{aligned}
& N_{t}^{M}=\int_{0}^{t} \psi_{M}\left(\tilde{V}_{s}^{M}\right) d s-\tilde{G}_{M}^{-1} \psi_{M}\left(\tilde{V}_{t}^{M}\right)+\widetilde{G}_{M}^{-1} \psi_{M}\left(\tilde{V}_{0}^{M}\right), \\
& X_{t}^{M}=\int_{0}^{t}\left(\phi_{M}-\psi_{M}\right)\left(\tilde{V}_{s}^{M}\right)-\widetilde{G}_{M}^{-1}\left(\phi_{M}-\psi_{M}\right)\left(\tilde{V}_{t}^{M}\right)+\widetilde{G}_{M}^{-1}\left(\phi_{M}-\psi_{M}\right)\left(\tilde{V}_{0}^{M}\right), \\
& Y_{t}^{M}=\widetilde{G}_{M}^{-1} \psi_{M}\left(\tilde{V}_{t}^{M}\right)-\widetilde{G}_{M}^{-1} \psi_{M}\left(\tilde{V}_{0}^{M}\right), \\
& Z_{t}^{M}=\widetilde{G}_{M}^{-1}\left(\phi_{M}-\psi_{M}\right)\left(\tilde{V}_{t}^{M}\right)-\tilde{G}_{M}^{-1}\left(\phi_{M}-\psi_{M}\right)\left(\tilde{V}_{0}^{M}\right) .
\end{aligned}
$$

We shall prove that if $1 \ll M(A) \ll A$, then

I. $\quad \frac{N_{A t}^{M(A)}}{\sqrt{A}} \Rightarrow W^{(\underline{\sigma})}$

by Theorem 5.1 of $\mathrm{H}(1982)$;

II. $\quad \frac{X_{A t}^{M(A)}}{\sqrt{A}} \Rightarrow 0$

by Doob's inequality;

III. $\quad \frac{Y_{A t}^{M(A)}}{\sqrt{A}} \Rightarrow 0$

as an indirect consequence of Lemma $1.12 \mathrm{~K}-\mathrm{V}$ (1986); and finally

IV. $\quad \frac{Z_{A t}^{M(A)}}{\sqrt{A}} \Rightarrow 0$

as a direct consequence of the same lemma. In what follows we give the basic ideas of the proof of steps I and III trying, however, to avoid calculational details. The proof 
of points II and IV is a straightforward calculation - here we give no details. We emphasize that, in the calculations, only $L_{2}$ manipulations and estimates are involved, relations (4.9), (4.10), and (4.11) being at any moment at hand.

I. The conditional variance process associated to the martingale $N^{M}$ [cf. $\mathrm{H}$ (1982)] is

where

$$
\left\langle N^{M}\right\rangle_{t}=\int_{0}^{t} \xi_{M}\left(\tilde{V}_{s}^{M}\right) d s
$$

$$
\xi_{M}=\left(\frac{\sigma^{2}}{2}\right)^{2} M^{2}\left(\widetilde{G}_{M}\left(\phi_{M}^{2}\right)-2 \phi_{M} \cdot \widetilde{G}_{M} \phi_{M}\right) \in \mathscr{H}_{M}
$$

Using the fact that $U^{M}(f \cdot g)=U^{M} f \cdot U^{M} g$, we have

$$
U^{M} \xi_{M}=\left(\frac{\sigma^{2}}{2}\right)^{2} \cdot\left(\left(M G_{M}\right)\left(\phi^{2}\right)-2 \phi \cdot\left(M G_{M}\right) \phi\right) \in \mathscr{H},
$$

and after some calculations one finds

$$
\left(U^{M} \xi_{M}\right)(x)=\left(\frac{\sigma^{2}}{2}\right)^{2} \cdot \frac{4 M^{2}}{(M+1)^{2}} \frac{1}{\sqrt{2 \pi}} \int d y e^{-\frac{y^{2}}{2}}\left|y-\frac{x}{\sqrt{M}}\right|^{3} .
$$

Hence

$$
\left(\mathbb{1}, \xi_{M}\right)_{M} \rightarrow \underline{\sigma}^{2} \text { and }\left\|\xi_{M}\right\|_{M}^{2}<\text { const. }
$$

Having these, it is an easy task to prove

$$
E\left(\frac{\left\langle N^{M(A)}\right\rangle_{A t}-A t \underline{\sigma}^{2}}{A}\right)^{2} \rightarrow 0
$$

Thus the conditional variance converges in probability to $\sigma^{2} \cdot t$. For applying Helland's theorem we have to estimate the largest jump of $N_{A t}^{M(\bar{A})}$, too. But this is an easy task because

$$
\frac{1}{\sqrt{A}} \sup _{t \leqq A}\left|\Delta N_{t}^{M(A)}\right|=\frac{\sigma^{2}}{2} \cdot \frac{M(A)}{\sqrt{A}} \sup _{t \leqq A}\left|\Delta \widetilde{V}_{t}^{M(A)}\right|
$$

and by standard arguments

$$
\operatorname{Prob}\left(M \cdot \sup _{0<t<1}\left|\Delta \tilde{V}_{t}^{M}\right|>c\right)<B e^{-\alpha c^{\nu}}
$$

with some positive constants $B, \alpha, \gamma$ (see the collision rules). Hence

$$
\frac{1}{\sqrt{A}} \sup _{t \leqq A}\left|\Delta N_{t}^{M(A)}\right| \rightarrow 0
$$

in any reasonable sense.

For obtaining III, first observe that, applying Lemma 1.12 of K-V (1986) to the function

$$
g_{M} \in \mathscr{H}_{M} ; \quad g_{M}(x)=\exp \sqrt{M}|x|
$$


we find

$$
\operatorname{Prob}\left(\sup _{t<M} \sqrt{M}\left|\widetilde{V}_{t}^{M}\right|>c\right) \leqq B \cdot e^{-c}
$$

with some positive constant $B$, independently of $M$. Since

$$
\begin{gathered}
Y_{t}^{M}=-\frac{\sigma^{2}}{2} M\left(\tilde{V}_{t}^{(M)}-\tilde{V}_{0}^{M}\right) \\
\operatorname{Prob}\left(\sup _{t \leqq A}\left|Y_{t}^{M(A)}\right| \geqq \eta \sqrt{A}\right) \\
\leqq 2 \operatorname{Prob}\left(\sup _{t \leqq A} \sqrt{M(A)}\left|\tilde{V}_{t}^{M(A)}\right| \geqq \eta^{\prime} \sqrt{\frac{A}{M(A)}}\right) \\
\leqq 2 \frac{A}{M(A)} \cdot \operatorname{Prob}\left(\sup _{t \leqq M(A)} \sqrt{M(A)}\left|\tilde{V}_{t}^{M(A)}\right| \geqq \eta^{\prime} \sqrt{\frac{A}{M(A)}}\right) \\
\leqq 2 B \frac{A}{M(A)} \cdot \exp -\eta^{\prime} \sqrt{\frac{A}{M(A)} \rightarrow 0} .
\end{gathered}
$$

(In the second inequality the stationarity of the process $\tilde{V}_{t}^{M}$ was used.) Modulo some calculational details, Theorem 3.1 is proved.

\section{The Coupling Lemma}

The present section consists of two subsections. In the first one we give the standard coupling of the mechanical and Markovian velocity processes $V_{t}^{M}$ respectively $\widetilde{V}_{t}^{M}$. In the second one we show that the two normalized position processes, $A^{-1 / 2} Q_{A t}^{M(A)}$ and $A^{-1 / 2} \widetilde{Q}_{A t}^{M(A)}$, realized in this way are sufficiently close to each other to produce the same asymptotic law, provided $M(A) \gg A^{\frac{1}{2}+\varepsilon}$. This result, combined with point (ii) of Theorem 3.1 proves Theorem 2.1.

5a. The coupling is, in principle, similar to that used in D-G-L (1981) (for their exposition, the reader should read Sect. 5 of that paper, too), but its realization here slightly deviates from theirs. We consider illuminating to give an intuitive phenomenological description rather than a very formalized one.

In the mechanical model, the process $V_{t}^{M}$ is driven by two mechanisms.

a) Markovian part: collisions with fresh light particles never seen in the past; and

b) non-Markovian part: recollisions. More exactly: let

$$
w_{t}^{M-}=\inf _{-\infty<s \leqq t} \frac{Q_{t}^{M}-Q_{s}^{M}}{t-s} ; \quad w_{t}^{M+}=\sup _{-\infty<s \leqq t} \frac{Q_{t}^{M}-Q_{s}^{M}}{t-s} .
$$

By Lemma 1 of Sz-T (1986), $w_{t}^{M \pm}$ are finite $\mu^{M}$-a.s. and

$$
\mu^{M}\left(\left\{\left|w_{t}^{M \pm}\right|>c\right\}\right)<\text { const } \exp \left(-\alpha(\sqrt{M} c)^{\beta}\right)
$$

for some positive constants $\alpha$ and $\beta$. The variables $w_{t}^{M \pm}$ and $V_{t}^{M}$ are measurable with respect to the $\sigma$-algebra $\mathscr{F}_{t}^{-}$generated by the past history of the Brownian particle 
$\left\{V_{s}^{M}:-\infty<s \leqq t\right\}$ and satisfy

$$
w_{t}^{M-} \leqq V_{t}^{M} \leqq w_{t}^{M+}
$$

The Markovian part of the driving mechanism consists of fresh light particles having velocities $v \notin\left[w_{t}^{M-}, w_{t}^{M+}\right]$ which hit the Brownian particle following a Poissonian law with instantaneous rate

$$
\varrho^{\operatorname{Mech}}\left(V_{t}^{M}, w_{t}^{M-}, w_{t}^{M+} ; v\right)=\frac{1}{\sqrt{2 \pi}} e^{-\frac{v^{2}}{2}}\left|V_{t}^{M}-v\right|_{\left[w_{t}^{M-}, w_{t}^{M+}\right]^{c}}(v) .
$$

After the collision - governed by the rules (2.1) - each light particle remains in the system waiting for possible recollisions.

The non-Markovian part consists of recollisions with old light particles which have velocities $v \in\left[w_{t}^{M-}, w_{t}^{M+}\right]$.

The description - in the same terms - of the driving mechanism of the Markov processes $\widetilde{V}_{t}^{M}$ was actually given in Sect. 3: fresh light particles collide with the Brownian particle following a Poissonian law with instantaneous rate

$$
\varrho^{\mathrm{Mark}}\left(\tilde{V}_{t}^{M} ; v\right)=\frac{1}{\sqrt{2 \pi}} e^{-\frac{v^{2}}{2}}\left|\tilde{V}_{t}^{M}-v\right|
$$

and recollisions are excluded.

For the coupling we should realize the two driving mechanisms jointly in such a way that the two processes suffer collisions with as many common light particles as possible.

For this reason let us define four instantaneous rates of incoming light particles. All of them depend on the variables $\left(V_{t}^{M}, \widetilde{V}_{t}^{M}, w_{t}^{M-}, w_{t}^{M+} ; v\right)$. Whenever possible, the notation of this dependence will be ignored.

$$
\begin{aligned}
& \varrho_{1}=\min \left(\varrho^{\operatorname{Mech}}\left(V_{t}^{M}, w_{t}^{M-}, w_{t}^{M+} ; v\right), \varrho^{\text {Mark }}\left(\tilde{V}_{t}^{M} ; v\right) \cdot \mathbb{1}_{\left[w_{t}^{M-}, w_{t}^{M+}\right]^{c}}(v)\right), \\
& \varrho_{2}=\varrho^{\text {Mech }}-\varrho_{1}, \\
& \varrho_{3}=\varrho^{\text {Mark }} \mathbb{1}_{\left[w_{t}^{M-}, w_{t}^{M+}\right]^{c}}(v)-\varrho_{1}, \\
& \varrho_{4}=\varrho^{\text {Mark }} \mathbb{1}_{\left[w_{t}^{M-}, w_{t}^{M+}\right]}(v) .
\end{aligned}
$$

The joint driving mechanism is described below.

1. Common Collisions. Fresh light particles of velocity $v \notin\left[w_{t}^{M-}, w_{t}^{M+}\right]$ hit simultaneously both Brownian particles in a Poissonian way with instantaneous rate $\varrho_{1}$.

2. Compensations. Fresh light particles - having $v \notin\left[w_{t}^{M-}, w_{t}^{M+}\right]$, too - come to the mechanical (respectively Markovian) Brownian particle following a Poissonian law with instantaneous rate $\varrho_{2}$ (respectively $\varrho_{3}$ ).

3. "Slow" Incoming Particles. a) Old light particles with $v \in\left[w_{t}^{M-}, w_{t}^{M+}\right]$ come to recollide with the mechanical Brownian particle.

b) Fresh light particles with $v \in\left[w_{t}^{M-}, w_{t}^{M+}\right]$ hit the Markovian Brownian particle following a Poissonian law with instantaneous rate $\varrho_{4}$.

We hope that after some meditation the reader will be convinced of the fact that the processes $V_{t}^{M}$ and $\widetilde{V}_{t}^{M}$ having initial values $V_{0}^{M}=\widetilde{V}_{0}^{M}$ distributed accord- 
ing to $d F^{M}$ and driven by the mechanism described above are exactly those which we need.

Without giving further formal details, we shall denote the probability space on which the coupled processes $\left(V_{t}^{M}, \widetilde{V}_{t}^{M}\right)$ are realized by $\left(\mathscr{Y}^{M}, P^{M}\right)$. (One can think of $\left(\mathscr{Y}^{M}, P^{M}\right)$ as $\mathscr{Y}^{M}=\mathfrak{X}^{M} \times \mathfrak{3}^{M}$ with $\mathfrak{3}^{M}$ being some measurable space and $P^{M}\left(B \times 3^{M}\right)=\mu^{M}(\beta)$.)

5. Closeness of the Paths. We shall prove the following

Lemma 5.1 (Coupling Lemma). If $M(A) \gg A^{\frac{1}{2}+\varepsilon}$, then for any $\eta>0$ and $t>0$

$$
P^{M(A)}\left(\left\{A^{-1 / 2} \int_{0}^{A t} d s\left|V_{s}^{M(A)}-\tilde{V}_{s}^{M(A)}\right|>\eta\right\}\right) \rightarrow 0 .
$$

Remark. The following inequalities are evident

$$
\begin{gathered}
P^{M(A)}\left(\left\{\sup _{0 \leqq t^{\prime} \leqq t} A^{-1 / 2}\left|\int_{0}^{A t^{\prime}} d s\left(V_{s}^{M(A)}-\widetilde{V}_{s}^{M(A)}\right)\right|>\eta\right\}\right) \\
\leqq P^{M(A)}\left(\left\{A^{-1 / 2} \int_{0}^{A t} d s\left|V_{s}^{M(A)}-\widetilde{V}_{s}^{M(A)}\right|>\eta\right\}\right) \\
\leqq P^{M(A)}\left(\left\{\sup _{0 \leqq t^{\prime} \leqq t} t A^{1 / 2}\left|V_{A t^{\prime}}^{M(A)}-\widetilde{V}_{A t^{\prime}}^{M(A)}\right|>\eta\right\}\right) .
\end{gathered}
$$

To have a "good coupling" i.e. to have (3.2), it is necessary and sufficient to show that the smallest probability of this chain converges to zero. Unfortunately, with our present method, we are able to handle only the second expression. As one can find after understanding the dynamics of the proof, Lemma 5.1 is sharp in this context; that is: if $M(A)=O(\sqrt{A})$, then the assertion of the lemma does not hold. (But the coupling may still be "good" - and we expect, actually it is "good" for $M(A) \gg 1$ !) On the other hand, for $M(A) \gg A^{\frac{3}{5}+\varepsilon}$, we are able to prove that the largest probability above still converges to zero (this fact may be useful if one also wants to bound the decay of the velocity autocorrelation function).

Proof of the Coupling Lemma. We shall consider $t=1$ and $M(A)$ a fixed function satisfying the condition of the Lemma. The proof will go as follows: we shall consider two large sets $\mathscr{B}_{A}, \mathscr{C}_{A} \subset \mathscr{Y}^{M(A)}$ with probabilities tending to one and a random process $\delta_{s}^{M(A)}, s \in[0, A]$ satisfying

$$
\delta_{s}^{M(A)} \geqq\left|V_{S}^{M(A)}-\tilde{V}_{S}^{M(A)}\right| \quad \text { on } \quad \mathscr{B}_{A} \cap \mathscr{C}_{A} .
$$

Finally we shall prove

Let

$$
\frac{1}{\sqrt{A}} \int_{0}^{A} d s \delta_{s}^{M(A)} \stackrel{\text { Prob. }}{\longrightarrow} 0
$$

and

$$
c(A)=[M(A)]^{-1 / 2} A^{\varepsilon} ; \quad d(A)=[M(A)]^{-1} \cdot A^{\varepsilon / 2}
$$

$$
\mathscr{B}_{A}=\left\{\sup _{0 \leqq s \leqq A}\left|V_{s}^{M(A)}\right|<c(A)\right\} \cap\left\{\sup _{0 \leqq s \leqq A}\left|\widetilde{V}_{s}^{M(A)}\right|<c(A)\right\} \cap\left\{\left|w_{0}^{M(A) \pm}\right|<c(A)\right\},
$$


$\mathscr{C}_{A}=\left\{\right.$ largest absolute jump of $V_{s}^{M(A)}$ or $\tilde{V}_{s}^{M(A)}$ in the interval

$$
s \in[0, A] \text { is less than } d(A)\} .
$$

By standard arguments we have $P^{M(A)}\left(\mathscr{B}_{A}\right) \rightarrow 1$, and $P^{M(A)}\left(\mathscr{C}_{A}\right) \rightarrow 1$. [In the proof, which we omit, of these assertions, the following facts are used: $\widetilde{V}_{S}^{M(A)}, V_{S}^{M(A)}$, and $w_{s}^{M(A) \pm}$ are stationary processes of typical order $\sim M(A)^{-1 / 2}$, and the jumps of $\tilde{V}_{s}^{M(A)}$ and $V_{s}^{M(A)}$ are of typical order $\sim M(A)^{-1}$, cf. the collision rules (2.1).]

For the further work we need a more detailed analysis of the driving mechanism.

1. Common collisions. From the collision rules (2.1), one finds that the effect on $V-\tilde{V}$ of a collision with a common light particle is

$$
V_{s^{+}}^{M(A)}-\tilde{V}_{s^{+}}^{M(A)}=\left(1-\frac{2}{M(A)+1}\right)\left(V_{s^{-}}^{M(A)}-\tilde{V}_{s^{-}}^{M(A)}\right) .
$$

A very important fact is that, on the set $\mathscr{B}_{A}$, the total instantaneous rate of common collisions satisfies for all $t \in[0, A]$,

$$
\bar{\varrho}_{1} \stackrel{\text { def }}{=} \int d v \varrho_{1} \geqq \alpha>0
$$

with $\alpha$ being a universal constant. One can check this relation by simple calculation using that, on $\mathscr{B}_{A},\left|w_{t}^{M(A) \pm}\right|<c(A)$ for all $t \in[0, A]$. Thus, this part of the mechanism has a contractive effect on $|V-\widetilde{V}|$, with constant rate.

2. Compensations. From the collision rules and the compensation rates we find that

$$
\operatorname{sign} A\left(V_{s}^{M(A)}-\tilde{V}_{s}^{M(A)}\right)=-\operatorname{sign}\left(V_{s^{-}}^{M(A)}-\tilde{V}_{s^{-}}^{M(A)}\right)
$$

for each jump caused by this part of the driving mechanism. (Here and in the sequel $\Delta f_{s}$ means the instantaneous jump of the function $f$ at the moment $s$.) Thus, collisions with compensational light particles either draw the two velocity processes closer to each other or change the sign of the difference process. Only the second effect can be harmful from our point of view. But on $\mathscr{C}_{A}$ the largest sign-changing jump of this kind is less than $A^{-(1+\varepsilon) / 2}$.

3. For collisions of the third kind, from the collision rules we have on $\mathscr{B}_{A}$,

$$
\left|\Delta V_{\mathrm{s}}^{M(A)}\right|<\frac{2}{M(A)}\left(\left|V_{\mathrm{s}^{-}}^{M(A)}\right|+\left|v^{-}\right|\right) \leqq \frac{4 c(A)}{M(A)}
$$

(and the same for $\left|\Delta \tilde{V}_{s}^{M(A)}\right|$ ).

Now we construct the majorizing process $\delta^{A}$ promised above. Let the process $\bar{\delta}^{A}$ be constructed in the following way: $\bar{\delta}_{s}^{A}$ is pure jump starting from zero $\bar{\delta}_{0}^{A}=0$, and driven by the following rules:

1. Whenever a jump of $V$ or $\tilde{V}$ caused by the third driving mechanism occurs (collisions with atoms coming with velocity $v \in\left[w_{t}^{M-}, w_{t}^{M+}\right], \bar{\delta}^{A}$ suffers an upwards jump

$$
\Delta \bar{\delta}_{s}^{A}=\frac{4 c(A)}{M(A)} .
$$

2. Whenever a common collision occurs, $\delta^{A}$ suffers a downwards jump

$$
\Delta \bar{\delta}_{s}^{A}=-\frac{2}{M(A)+1} \bar{\delta}_{s^{-}}^{A} .
$$


Let

$$
\delta_{s}^{A}=A^{-(1+\varepsilon) / 2}+\bar{\delta}_{s}^{A} .
$$

It is an easy task to check that (5.2) is preserved after each collision.

Assertion (5.3) remains to be proved. In fact we shall prove

if $M(A) \gg A^{\frac{1}{3}+\varepsilon}$.

$$
\frac{1}{\sqrt{A}} \int_{0}^{A} d s \bar{\delta}_{s}^{A} \stackrel{\text { Prob. }}{\longrightarrow} 0
$$

The germ of the forthcoming proof is the following picture valid on $\mathscr{B}_{A}$ : assume, for simplicity, that $M(A)=A^{\gamma} ; \bar{\delta}_{s}^{A}$ is composed of two effects: additions of order $A^{\varepsilon-\frac{3 \gamma}{2}}$ with "average" rate below $O\left(A^{2 \varepsilon-\gamma}\right)$ and contractive multiplications by $\frac{M(A)-1}{M(A)+1} \sim 1-\frac{2}{A^{\gamma}}$ with rate uniformly above $\alpha>0$ (see (5.4)). The evolutions of the additive terms can, of course, be separated and, as a consequence of the fact that contractions occur sufficiently uniformly, they decay exponentially resulting that their sum is $o\left(A^{1 / 2}\right)$ only, provided $\gamma>\frac{1}{3}+\varepsilon$.

Let $\tau_{i}$ be the moments of upwards jumps of the process $\bar{\delta}_{s}^{A}$ in the time interval $s \in[0, A]$,

where

$$
\#\left\{\tau_{i}\right\}=N_{1}^{A}+N_{2}^{A},
$$

$N_{1}^{A}=$ number of recollisions in $[0, A]$,

$N_{2}^{A}=$ number of collisions of the third type of the Markovian process in $[0, A]$.

Further let

$$
N_{3}^{A}[s, t]=\text { number of common collisions in }[s, t] \text {. }
$$

We have

$$
\bar{\delta}_{s}^{A}=\frac{4 c(A)}{M(A)} \cdot \sum_{i: s \geqq \tau_{\imath}}\left(1-\frac{2}{M(A)+1}\right)^{N_{3}^{A}\left[\tau_{i}, s\right]} .
$$

Thus

$$
\begin{aligned}
\int_{0}^{A} d s \bar{\delta}_{s}^{A} & =\frac{4 c(A)}{M(A)} \cdot \sum_{i} \int_{\tau_{i}}^{A} d s\left(1-\frac{2}{M(A)+1}\right)^{N_{3}^{A}\left[\tau_{i}, s\right]} \\
& \leqq \frac{4 c(A)}{M(A)} \cdot\left(N_{1}^{A}+N_{2}^{A}\right) \cdot \sup _{0 \leqq t \leqq A} \int_{t}^{A} d s\left(1-\frac{2}{M(A)+1}\right)^{N_{3}^{A}[t, s]} .
\end{aligned}
$$

On the set $\mathscr{B}_{A}$,

$$
N_{i}^{A} \leqq \bar{N}_{i}^{A}, \quad i=1,2,
$$

where $\bar{N}_{1}^{A}\left(\bar{N}_{2}^{A}\right)$ is the number of collisions of the mechanical (Markovian) Brownian particles with incoming light particles having velocity less than $c(A)$, and

$$
P^{M(A)}\left(A^{\frac{\varepsilon}{100}} c(A) \bar{N}_{i}^{A}>\eta \sqrt{A}\right) \leqq \frac{c(A) A^{\frac{\varepsilon}{100}}}{\eta \sqrt{A}} E \bar{N}_{i}^{A}=\frac{\text { const }}{\eta} \cdot A^{\frac{\varepsilon}{100}} c(A)^{3} \sqrt{A}
$$


Thus

$$
P^{M(A)}\left(\frac{A^{\frac{\varepsilon}{100}} c(A)\left(N_{1}^{A}+N_{2}^{A}\right)}{\sqrt{A}}>\eta\right) \rightarrow 0,
$$

provided $M(A) \gg A^{\frac{1}{3}+\varepsilon}$. On the other hand, by (5.4), on $\mathscr{B}_{A}$,

$$
N_{3}^{A}[t, s] \geqq \bar{N}_{3}[t, s],
$$

where $\bar{N}_{3}[t, s]$ is a Poissonian process with constant rate $\alpha$, and hence

$$
\begin{aligned}
\int_{t}^{A}\left(1-\frac{2}{M(A)+1}\right)^{N_{3}^{A}[t, s]} d s & <\int_{t}^{A} d s\left(1-\frac{2}{M(A)+1}\right)^{\bar{N}_{3}[t, s]} \\
& <\int_{t}^{\infty} d s\left(1-\frac{2}{M(A)+1}\right)^{\bar{N}_{3}[t, s]} \stackrel{\text { def }}{=} Y_{t}^{M(A)} .
\end{aligned}
$$

$Y_{t}^{M(A)}$ is a stationary random process depending in a relatively simple way on the Poisson process $\bar{N}_{3}$. By some standard arguments concerning Poisson processes it is not hard to show that

$$
\frac{1}{A^{\frac{\varepsilon}{100}}} \sup _{0<t<A}\left(\frac{1}{M(A)} Y_{t}^{M(A)}\right) \stackrel{\text { Prob. }}{\longrightarrow} 0 .
$$

We omit the details of this step. Now by combining (5.6) and (5.7), (5.5) is obtained, and hence the proof of the lemma.

Acknowledgements. It is a pleasure to thank József Fritz and Bernard Souillard for illuminating discussions on the functional analytic problems involved and Shelley Goldstein for detailed comments on the first version of the paper. B. T. also thanks the kind hospitality of the Institute for Mathematics and its Applications, Minneapolis, where part of this work was done.

\section{References}

B (1968) Billingsley, P.: Convergence of probability measures. New York: Wiley 1968

B (1788) Bürger, G.A.: Des Freiherrn von Münchhausen Reisen und Abenteuer zu Wasser und Lande, wie er dieselben bei einer Flasche im Zirkel seiner Freunde zu erzählen pflegte. Göttingen, $\times 1788$

D-G (1986) Dürr, D., Goldstein, S.: Remarks on the central limit theorem for weakly dependent random variables. In: Stochastic processes - Mathematics and physics. Albeverid, S., Blanchard, P., Streit, L. (eds.). Lecture Notes in Mathematics, Vol. 1158. Berlin, Heidelberg, New York: Springer 1986

D-G-L (1981) Dürr, D., Goldstein, S., Lebowitz, J.L.: A mechanical model of Brownian motion. Commun. Math. Phys. 78, 507-530 (1981)

G-G (1986) Goldstein, S., Guetti, J.: On the diffusion of the fast molecule. J. Stat. Phys. (to appear)

G-L (1978) Gordin, M.I., Lifshic, B.A.: Central limit theorem for stationary Markov chains. Dokl. Akad. Nauk. SSSR 239, 766-767 (1978)

H (1982) Helland, I.: Central limit theorems for martingales with discrete or continuous time. Scand. J. Stat. 9, 79-94 (1982)

H (1965) Harris, T.E.: Diffusions with collisions between particles. J. Appl. Probab. 2, 323-338 (1965) 
H (1971) Holley, R.: The motion of a heavy particle in an infinite one dimensional gas of hard spheres. Z. Wahrscheinlichkeitstheor. Verw. Geb. 17, 181-219 (1971)

K-V (1986) Kipnis, C., Varadhan, S.: A central limit theorem for additive functionals of reversible Markov processes and applications to simple exclusion. Commun. Math. Phys. 104, 1-19 (1986)

N (1967) Nelson, E.: Dynamical theory of Brownian motion. Princeton, NJ: Princeton University Press 1967

N (1964) Neveu, J.: Bases mathématiques du calcul des probabilités. Paris: Masson 1964

O-R-D (1985) Omerti, E., Ronchetti, M., Dürr, D. : Numerical evidence for mass dependence in the diffusive behaviour of the "Heavy Particle" on the line. J. Stat. Phys. 44, 339-346 (1986)

R-S (1978) Reed, M., Simon, B.: Methods of modern mathematical physics. IV. Analysis of operators. New York: Academic Press 1978

S (1986) Sinai, Ya.G.: Oral communication

S-S (1986) Sinai, Ya.G., Soloveichik, M.R.: One-dimensional classical massive particle in the ideal gas. Commun. Math. Phys. 104, $423-443$ (1986)

S (1969) Spitzer, F.: Uniform motion with elastic collisions of an infinite particle system. J. Math. Mech. 18, 973-989 (1969)

Sz-T (1986) Szász, D., Tóth, B.: Bounds on the limiting variance of the heavy particle. Commun. Math. Phys. 104, 445-455 (1986)

Communicated by J. L. Lebowitz

Received June 4, 1986; in revised form January 16, 1987

\section{Notes added in proof.}

1. In the corresponding multidimensional model the Brownian particle is spherical having radius $R$. Here the cases when $M(A) \rightarrow \infty$ are treatable in an analogous way as for $d=1$, with some additional geometrical considerations involved. In the results $R$ enters trivially as a rescaling factor $R^{d-1}$ of the time parameter of the limiting process. [However, this is no more true for $M(A)=0(1)$.] Case 5 (in the diffusion scaling) is trivial, too. Case 4 was treated in D-G-L (1981). Finally, a complete analogue of Theorem 2.1 can be proved with the limiting variance being $\sigma_{R}^{2}=R^{1-d} d \cdot \sqrt{\frac{\pi}{8}}$

2. Concerning the super-heavy Brownian particle (Case $5: M(A) \gg A, d=1$ ), by an application of a theorem of Kurtz, [essentially the same method as that used in H(1971)], we find the correct asymptotics

$$
V_{A t}^{M(A)}=\frac{1}{\sqrt{M(A)}}\left(\sqrt{M(A)} V_{0}^{M(A)}\right)+\frac{\sqrt{A}}{M(A)} \xi_{t}^{A},
$$

where $\sqrt{M(A)} V_{0}^{M(A)}$ has a standard Gaussian distribution and $\xi_{t}^{A}$ converges in distribution to a Wiener process of variance $4 \sqrt{\frac{2}{\pi}}$. If $d>1$, a similar statement holds with a limiting variance depending on the dimension. 